\title{
Carbon nanotube fibers with martensite and austenite Fe residual catalyst: room temperature ferromagnetism and implications for CVD growth
}

Belén Alemán ${ }^{\mathrm{a}}$, Rocío Ranchal ${ }^{\mathrm{b}}$, Víctor Reguero ${ }^{\mathrm{a}}$, Bartolomé Mas ${ }^{\mathrm{a}}$ and Juan J. Vilatela ${ }^{\mathrm{a}, *}$

a IMDEA Materials Institute, Eric Kandel 2, 28906 - Getafe, Madrid, Spain

${ }^{\mathrm{b}}$ Dep, Física de Materiales, Universidad Complutense de Madrid, 28040 - Madrid, Spain

\begin{abstract}
We report on the room temperature ferromagnetic properties of continuous macroscopic fibers made up of carbon nanotubes grown by floating catalyst chemical vapor deposition. Their ferromagnetic behavior originates from the presence of residual catalyst nanoparticles: martensite with 0.77 wt. \% C content and FCC Fe. The first is intrinsically ferromagnetic, but the later only due to severe lattice distortion as a consequence of $\mathrm{C}$ supersaturation. The stabilization of martensite and austenite occurs mainly because of the small diameter of the nanoparticles, in the range of $4-20 \mathrm{~nm}$. This is smaller than the embryonic nucleus of the relevant equilibrium phases, but also implies that large $\mathrm{C}$ concentrations can build up in FCC Fe before $\mathrm{C}$ can be segregated as a stable graphitic nucleus. Room temperature remanence ranges from $10 \%$ to $25 \%$ and coercivity from 55 to $300 \mathrm{Oe}$, depending on the choice of promoter for fiber synthesis ( $\mathrm{S}$ or $\mathrm{Se}$ ). Superparamagnetic behavior is only observed in S-grown samples on account of the smaller diameter of residual catalysts particles. The results of this work provide an explanation for widespread observation of magnetic properties in oxide-free CNT samples produced by catalytic growth under a wide range of synthesis conditions.
\end{abstract}

\section{Introduction}

Transition metals like Fe, Co and Ni are widely used as catalyst for CNT growth by chemical vapor deposition (CVD). They present few unfilled d-orbitals that thus limit carbon solubility and enhance the formation of graphitic C [1]. CVD-grown 
samples of CNTs have a considerable fraction of residual catalyst, often higher than 30 wt. \%, consisting of distributed nanoparticles separated by the CNTs. These nanoparticles can be magnetic depending on their crystal structure [2] and they are encapsulated either inside the nanotubes or spherical carbon shells, in both cases preventing oxidation and providing magnetic separation between vicinal particles or magnetic structures [3]. Bulk samples of CNTs have hence been considered magnetically functionalized (MFCNT) and suitable for a range of potential applications in magnetoelectronics and biomedicine [4].

The interest in applications that exploit the presence of residual magnetic particles in CNT samples has often implied that the origin of such magnetic behavior is not studied in detail. Furthermore, explaining the formation of magnetic phases as a result of the CVD reaction has proven challenging. Ferromagnetism has been reported in CNT samples produced with Fe [5,6] or Co [7]. In particular, growth of CNTs under conventional $\mathrm{CVD}$ conditions at $700-900^{\circ} \mathrm{C}$ using $\mathrm{Fe}$ catalyst produces $\mathrm{FCC}, \mathrm{BCC}$ and cementite $\left(\mathrm{Fe}_{3} \mathrm{C}\right)$ crystal phases [8] and the observed ferromagnetism has been generally attributed to $\mathrm{BCC} \mathrm{Fe}$ or cementite $\left(\mathrm{Fe}_{3} \mathrm{C}\right)$ [3,9-11] since bulk FCC $\mathrm{Fe}$ is paramagnetic [12]. But some systems with distorted FCC Fe can in fact be ferromagnetic. Thin FCC Fe layers epitaxially grown on $\mathrm{Cu}(100)$ [13-15] develop perpendicular magnetic anisotropy due to lattice mismatch with the substrate and the resulting strain in the layer [13]. Room temperature ferromagnetism has also been claimed in FCC Fe catalyst nanoparticles produced from CNT synthesis [16]. This is attributed to the presence of C in FCC Fe structure increasing its lattice parameter and to a charge transfer between $\mathrm{C}$ and $\mathrm{Fe}$.

The examples of magnetic CNT samples discussed above include most common catalysts ( $\mathrm{Fe}$ and $\mathrm{Co}$ ), and include phases that are paramagnetic in the bulk. The ferromagnetic behavior in CNT samples would thus seem inevitable. But it remains to clarify how CNT growth conditions produce different phases of the same transition metal, what stabilizes metastable phases and what the $\mathrm{C}$ content in the nanoparticles is, amongst several questions. Their answer would provide a better understanding of CNT growth by CVD while also providing a tool to engineer magnetic properties by catalyst control.

In this work we provide evidence of ferromagnetic behavior in samples consisting of macroscopic fibers of CNTs produced by floating catalyst CVD. The residual catalyst had been previously shown to contain negligible amounts of BCC or 
cementite [17]. Here, we demonstrate that room temperature ferromagnetism in CNT fibers is due to the presence of martensite (M) and distorted FCC Fe residual catalyst nanoparticles. The stabilization of both phases is discussed in the context of the estimated cooling rates and on the barriers for nucleation of stable $\mathrm{Fe}-\mathrm{C}$ phases. Finally, we show that superparamagnetic properties correlate well with the observed catalyst particle size distribution obtained under different synthesis conditions.

\section{Experimental section}

\subsection{CNT fiber samples preparation}

CNT fibers (CNTfs) were synthesized by direct spinning of CNTs from the gas phase by floating catalyst CVD [18] using butanol as C source, ferrocene as Fe catalyst and S (S-CNTf) or Se (Se-CNTf) as promoters. The concentration of precursors during fiber production was adjusted so as to produce CNTs of predominantly single-wall (SWCNTs) or multi-wall (MWCNTs) of few layers $(<5)[19,20]$. Thus, four types of fibers were produced and analyzed: SWCNT from S, SWCNT from Se, MWCNT from S and MWCNT from Se (Table 1).

\subsection{CNT fiber samples characterization}

Thermogravimetric analysis (TGA) was carried out with a Q800 TA Instruments with a ramp of $10^{\circ} \mathrm{C} / \mathrm{min}$.

Catalyst crystal structure was identified by X-ray diffraction (XRD) and high resolution transmission electron microscopy (HRTEM). XRD data was acquired with an Empyrean PANalytical diffractometer, with $\mathrm{Cu} \mathrm{K} \alpha$ radiation $(1.54 \AA)$ and $45 \mathrm{kV} / 40$ $\mathrm{mA}$. For these measurements, CNT fiber sample was collected for 1.5 hours in order to maximize X-ray scattering intensity. Data acquisition was adjusted to enhance angular resolution, with $0.03^{\circ}$ step and $35 \mathrm{~s} / \mathrm{step}$ acquisition (13 hours measurement) for the full range pattern and $0.02^{\circ}$ and $4 \mathrm{~s} / \mathrm{step}$ acquisition (6 hours measurement) for the $40-50^{\circ} 2 \theta$

region. Data were analyzed after linear background subtraction and smoothing of the patterns with High Score software plus. HRTEM was performed with a JEOL JEM 3000F TEM at $300 \mathrm{kV}$ and catalyst structure was determined from the Fast-FourierTransform (FFT) of lattice-resolved micrographs.

\subsection{Magnetic measurements}


Hysteresis loops with applied magnetic field at different angles with respect to the fiber axis were measured at room temperature in a vibrating sample magnetometer (VSM) from LakeShore. The magnetic characteristics as a function of the temperature were probed with field-cooled (FC) and zero-field-cooled (ZFC) magnetization curves in a superconducting quantum interference device (SQUID) magnetometer. For FC curves, the sample was first cooled from room temperature to $5 \mathrm{~K}$ under a saturation field of $5 \mathrm{kOe}$ and then, the magnetization was recorded with an applied magnetic field of 100 Oe during heating. The same procedure was performed for the ZFC except that cooling was done in a zero applied magnetic field. Hysteresis loops at $10 \mathrm{~K}$ after FC and ZFC procedures were also recorded in the SQUID. In order to rule out spurious contributions to the room temperature ferromagnetic behavior of the fibers, we also measured hysteresis loops at $300 \mathrm{~K}$ in the SQUID magnetometer mounted differently from when measuring in the VSM. In addition, different pieces of the samples were measured in each magnetometer but hysteresis loops were identical in both cases (S1).

\section{Results and discussion}

\subsection{Room temperature ferromagnetism}

All the CNT fibers grown present ferromagnetism at low $(10 \mathrm{~K})$ and room temperature independently from the choice of promoter ( $\mathrm{S}$ or $\mathrm{Se}$ ) as indicated by the hysteresis and remanence loops in magnetic measurements at these two temperatures (Fig. 1a and S1). The hysteresis loops are not affected by the direction of the applied magnetic field (Fig 1b), therefore the ferromagnetic behavior exhibited by the fibers is considered as isotropic. Because the CNTs in the fiber are predominantly oriented along its main axis, the absence of anisotropy in the hysteresis loops indicates that the residual catalyst particles are the source of the magnetic behavior observed and discards any relevant contribution from the nanotubes. 

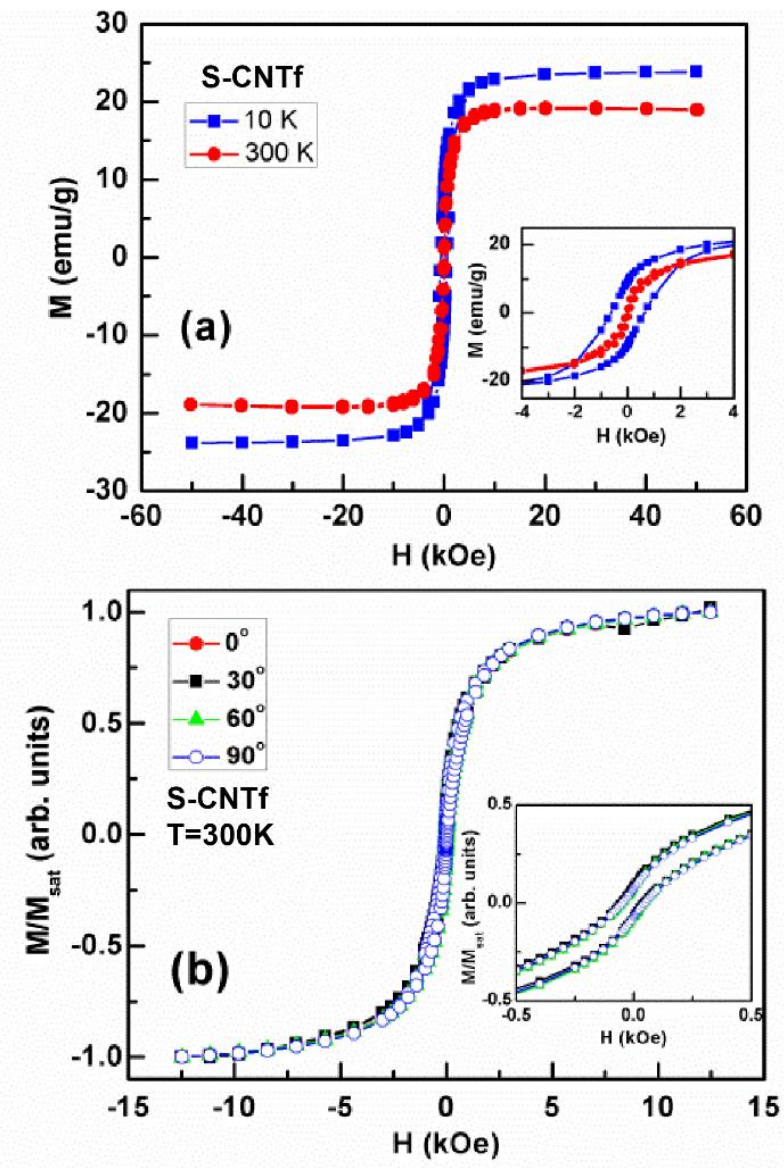

Fig. 1. (a) Room (•) and low temperature ( $\bullet$ SQUID hysteresis loops of S-CNT fiber confirming the presence of ferromagnetism. (b) Comparison of room temperature hysteresis loops of the sample in a VSM magnetometer for different orientations between the applied magnetic field and the fiber axis: $(\bullet)$ $0^{\circ}$, i.e. the applied magnetic field is in the direction of the fiber axis, ( $\left.\mathbf{\square}\right) 30^{\circ},(\boldsymbol{\Delta}) 60^{\circ}$ and (o) $90^{\circ}$, i.e. the applied magnetic field is perpendicular to the fiber axis.

In order to rule out the presence of antiferromagnetic phases, reported in CNTs synthesized by pyrolysis of similar precursors [21], we compared field-cooled and zero field-cooled loops recorded at $10 \mathrm{~K}$ and also, low and room temperature hysteresis loops of the same fiber (Fig. 1a). The absence of a shift in the field axis discards interactions between ferro- and antiferromagnetic phases and suggests a high homogeneity of magnetic phases in the CNT fibers of this study.

A summary of the coercivity (Hc) and saturation magnetization (Ms) of all the studied fibers is shown in Table I. At $10 \mathrm{~K}$ values for remanence are around $40-45 \%$ in all the fibers and coercivity ranges from $1.1 \mathrm{kOe}$ to $0.6 \mathrm{kOe}$ depending on the promoter. At $300 \mathrm{~K}$ there are substantial differences between the samples produced with different promoters, remanence and coercivity are 16-25\% and 200-300 Oe for samples grown 
with Se, compared with $10 \%$ and 55-70 Oe for those grown with S. Again, the differences in magnetic properties are ascribed to residual catalyst, with no effect of the CNT type other than in the conditions used for synthesis. For samples produced with different promoters the values of room temperature coercivity does not correlate well with residual Fe content determined by TGA ( $\mathrm{S} 2$ and Table 1). This is partly due to differences in particle size, shape [22] or the presence of defects, all of which have an impact on absolute values of coercivity.

\begin{tabular}{|lcccc|}
\hline Sample & \multicolumn{2}{c}{ S-CNTf } & \multicolumn{2}{c|}{ Se-CNTf } \\
\hline CNT type & SWNT & MWNT & sWNT & MWNT \\
Promoter/C [at.\%] & 0.04 & 0.33 & 0.01 & 0.10 \\
Fe [wt. \%] & 15 & 4 & 8 & 6 \\
Ms 300 K [emu/g] & 19 & 3 & 10 & 18 \\
Hc 300 K [Oe] & 70 & 55 & 300 & 198 \\
Ms 10 K [emu/g] & 24 & 4 & 11 & 21 \\
Hc 10 K [Oe] & 600 & 800 & 1100 & 1000 \\
$T_{B}[\mathrm{~K}]$ & 140 & 140 & $>\mathrm{RT}$ & $>\mathrm{RT}$ \\
\hline
\end{tabular}

Table 1. Summary of magnetic properties of samples composed different CNT type, and produced with different promoters. Data include: promoter to carbon ratio in precursor mixture, residual Fe wt. \% obtained by TGA and magnetic properties of the samples.

\subsection{Effect of particle size}

To further characterize the magnetic behavior of CNT fibers, FC-ZFC curves were also performed (Fig. 2 and Fig. S3). The distance between FC and ZFC curves at $300 \mathrm{~K}$ confirms the ferromagnetic behavior measured at room temperature by VSM and SQUID for all fibers. The fact that FC curves are not flat indicates the absence of a strong interparticle interaction [10], in this case prevented by graphitic encapsulation of the nanoparticles and the presence of the CNTs. It suggests that this material is well suited for applications requiring small separated magnetic domains [22]. 

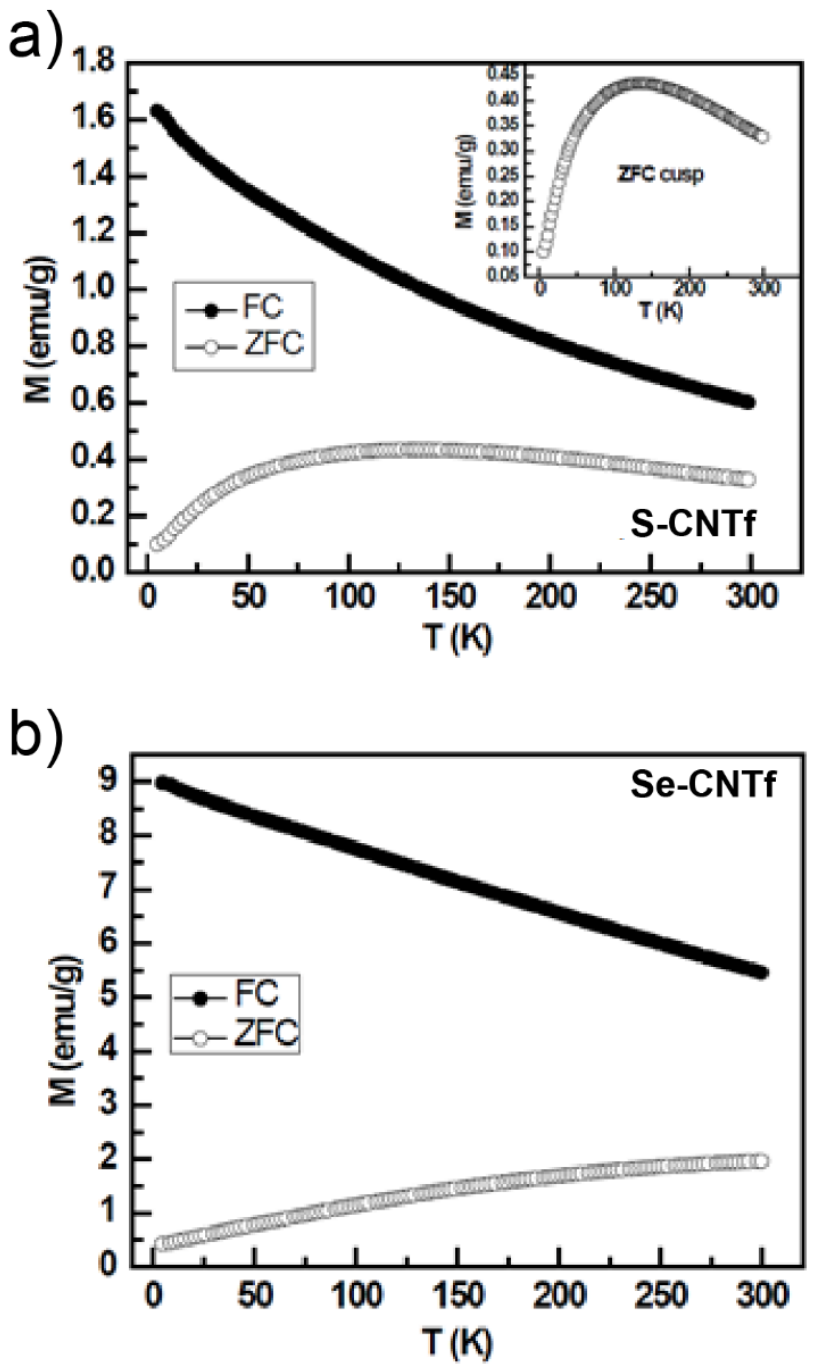

Fig. 2. FC-ZFC curves for (a) S- and (b) Se-CNT fibers. S-CNT fibers show clear superparamagnetic behaviour, with a $\mathrm{T}_{\mathrm{B}}$ around $140 \mathrm{~K}$, indicative of the presence of small nanoparticles.

The main difference between samples obtained from different promoters is the presence of a maximum (or a cusp) in the ZFC curves for fibers produced with $\mathrm{S}$, indicative of superparamagnetic behavior. Superparamagnetic behavior is a size dependent effect that occurs in nanoscopic materials, for which a transition from ferromagnetic to superparamagnetic takes place at a critical temperature, known as the blocking temperature $\left(\mathrm{T}_{\mathrm{B}}\right)$. The broad cusp in the ZFC (Fig 2a inset) reflects a wide range of superparamagnetic particle sizes and gives a blocking temperature $T_{B}$ of $140 \mathrm{~K}$. In contrast, ZFC curves for samples grown with Se show a continuous increase of the magnetization as the temperature is raised from 5 to $300 \mathrm{~K}$ (Fig. 2b). This corresponds to particles without a clear superparamagnetic behavior because of a blocking temperature higher than $300 \mathrm{~K}$ and/or an extremely wide size distribution. 
These results agree with the size distribution of catalyst particles obtained experimentally by extensive HRTEM analysis, presented in Fig. 3. The distribution of nanoparticles produced with $\mathrm{S}$ peaks at around 4-8 $\mathrm{nm}$ (Fig. 3a), whereas the distribution of produced with Se is centred at diameters around 9-15 nm (Fig. 3b). For the latter, as a consequence of such a relatively large particle size superparamagnetism is not observed at or below $300 \mathrm{~K}$. This can be confirmed by comparison with the theoretical particle size obtained from $\mathrm{T}_{\mathrm{B}}$ using equation (1):

$$
K V=k_{B} T_{B}
$$

where $K$ is the magnetic anisotropy constant, $V$ is the nanoparticle volume and $\mathrm{k}_{\mathrm{B}}$ is Boltzmann's constant. We consider the magnetic anisotropy constant $K_{\mathrm{Fe}_{-} B C C}=4.18 \times$ $10^{4} \mathrm{~J} / \mathrm{m}^{3}$ for $\mathrm{BCC} \mathrm{Fe}$, since to our knowledge there are no literature data on the anisotropy constant for metastable phases of Fe. Therefore a $T_{B}=140 \mathrm{~K}$ corresponds to a nanoparticle diameter around $4.5 \mathrm{~nm}(\mathrm{~S} 4)$, which is in the range determined by TEM for the superparamagnetic S-CNT fiber (Fig 3a) and in good agreement with experimental observations of superparamagnetism in BCC Fe encapsulated in CNTs [24]. For reference, $T_{B}=300 \mathrm{~K}$ gives a particle diameter of $6 \mathrm{~nm}$ (S4), from which it is clear that only the smaller nanoparticles in the sample contribute to the superparamagnetic behavior observed, while also confirming that the particle size in Se samples is too large to provide a clear superparamagnetic signal. 


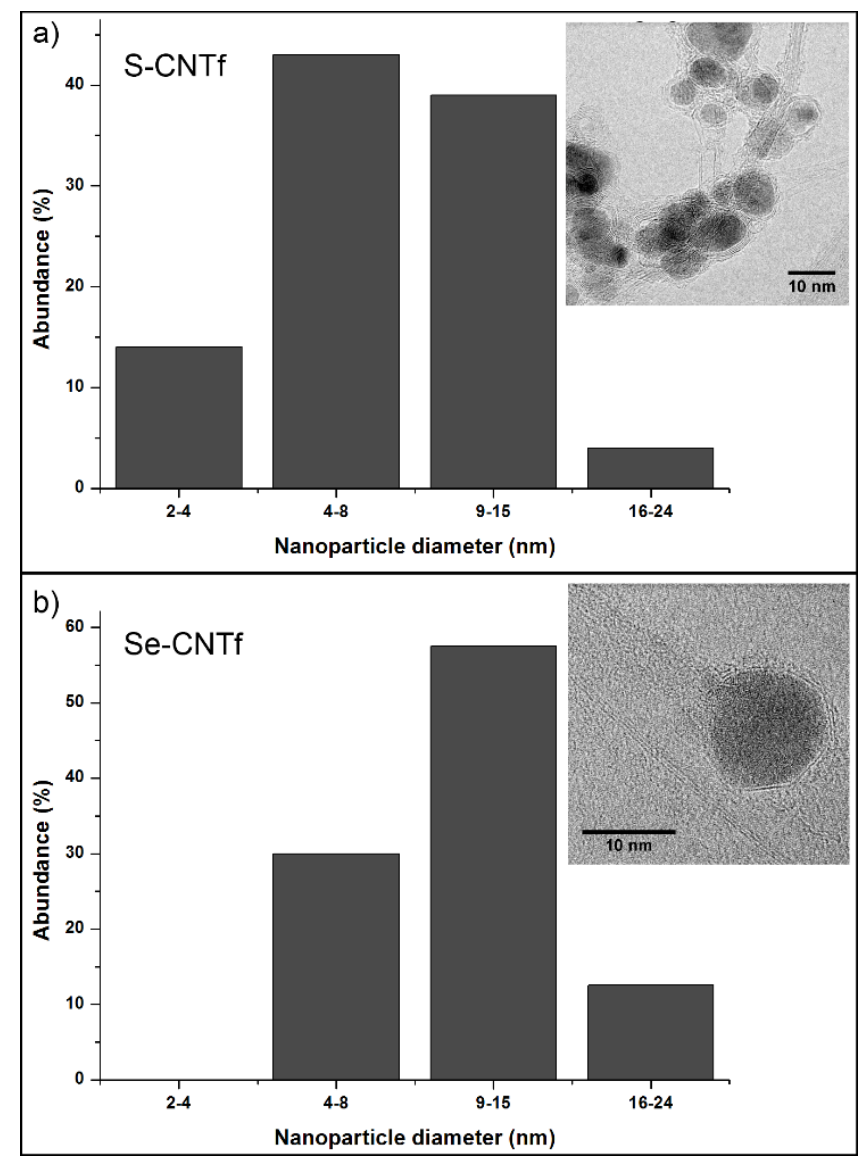

Fig. 3. Catalyst nanoparticle diameter distribution for (a) S- and (b) Se-CNTf, extracted from HRTEM images. Insets: examples of TEM micrographs of residual catalyst nanparticles.

\subsection{Origin of ferromagnetic behavior}

The results presented above clearly establish that the magnetic properties of CNT fibers are due to residual catalyst nanoparticles and that these are sensitive to their size distribution. It is then of interest to analyze the origin of the size-independent ferromagnetic behavior observed. We first note that of the 4-15 wt. \% residual Fe catalyst in the fibers more than $99.9 \%$ in fact does not lead to the growth of CNTs, but ends up trapped between CNT bundles as residual quasispherical catalyst particles capped by graphitic layers [19] (Fig. 3 insets). In previous studies the presence of Fe oxide was ruled out through a combination of X-ray photoelectron spectroscopy, HRTEM and elemental analysis. No evidence of BCC Fe or carbides (e.g. $\mathrm{Fe}_{3} \mathrm{C}$ ) was observed, and thus the residual catalyst was assigned mainly to FCC Fe [19,25]. XRD data obtained by azimuthal integration of 2D wide-angle X-ray patterns confirmed these observations, but was not of sufficient resolution to provide further information while 
also covering such a wide Q-range. Here we present new powder X-ray data collected under conditions optimized to analyze the catalyst region (see experimental section) and which help to explain the ferromagnetic behavior of CNT fibers discussed above.

Fig. 4a shows a XRD pattern in the range $40^{\circ}-80^{\circ}$, with intense reflections from the CNTs and evidence of the (200) and (220) reflections from FCC Fe. But interestingly, closer inspection of the patterns shows the presence of martensite. In a detailed plot in the range $40^{\circ}-50^{\circ}$ (Fig. 4b) the martensite (101) (110) reflections can now be clearly identified and distinguished from other intense contributions from FCC (111), CNTs (100) and the expected positions for $\mathrm{Fe}_{3} \mathrm{C}(031)(112)$. The split of the martensite (002) and (200) peaks at $62.7^{\circ}$ and $65.3^{\circ}$ respectively [26], is also observed. In addition to exposing the presence of martensite, the data confirm the absence of BCC $\mathrm{Fe}$ or Fe oxide. 


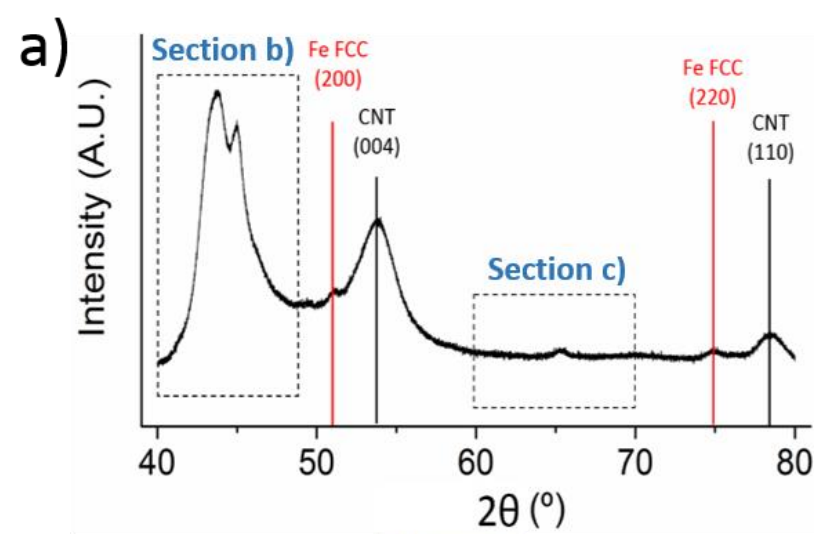

b)

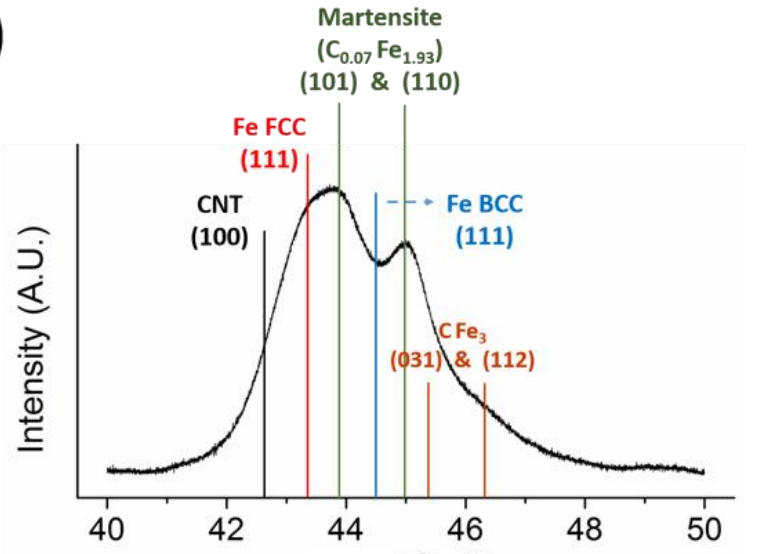

c)

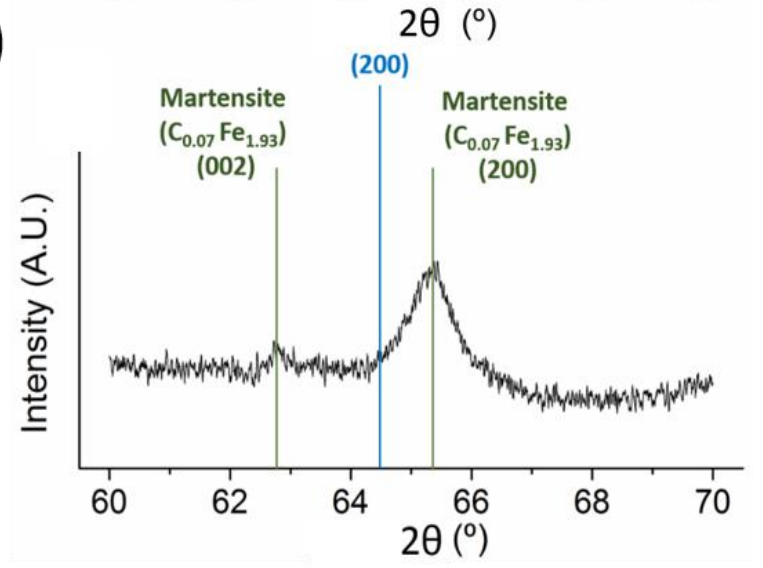

Fig. 4. XRD patterns showing the composition of CNT fibres. In the range (a) from $40^{\circ}$ to $80^{\circ}$, the presence of FCC Fe and graphitic peaks related to CNTs are clearly observed. Magnified data in the ranges of (b) $40^{\circ}-50^{\circ}$ and (c) $60^{\circ}-70^{\circ}$ clearly show the presence of FCC Fe and martensite with 0.77 wt. $\% \mathrm{C}$ as the major constituents of the catalyst, while confirming absence of BCC Fe.

The amount of interstitial carbon in martensite can be conveniently determined from the position of the XRD peaks, since the change in lattice parameters depends linearly on carbon content [27], related by equation (2):

$$
\mathrm{c} / \mathrm{a}=1+0.045 \mathrm{C}_{\mathrm{wt} .} \%
$$


From the (200) and (002) XRD peaks we obtained interplanar distances of 1.48 $\AA$ and $1.43 \AA$, giving lattice parameters of $\mathrm{a}=2.86 \AA$ and $\mathrm{c}=2.96 \AA$. The resulting carbon content $\mathrm{C}_{\mathrm{wt} . \%}$ comes out as $0.77 \mathrm{wt} \%$.

Martensite is ferromagnetic [28] and is thus largely responsible for the ferromagnetic behavior discussed above (Fig.1a, Fig. S1b and Fig. S1c further rule out contribution from $\mathrm{Fe}$ oxide $)^{1}$. But martensite alone cannot explain the observed high values of saturation magnetization. Therefore the implication is that $\mathrm{FCC} \mathrm{Fe}$ also contributes to ferromagnetic behavior of the samples. While retained austenite (FCC Fe) in bulk steel samples is paramagnetic [29], changes in FCC lattice spacing of Fe nanoparticles due to $\mathrm{C}$ supersaturation lead to ferromagnetic behavior [16]. Theoretical calculations suggest that interstitial C stabilizes FCC Fe and increases its magnetic moment (from $0 \mu_{\mathrm{B}}$ ) as a result of lattice expansion, reaching values around $2 \mu_{\mathrm{B}}$ for a lattice parameter increase of $0.03 \mathrm{~nm} \mathrm{[16].} \mathrm{Thus,} \mathrm{the} \mathrm{development} \mathrm{of} \mathrm{a} \mathrm{magnetic}$ moment occurs both as a consequence of lattice distortion and charge transfer between Fe and $\mathrm{C}$, since the two are in fact closely related.

Although it is difficult to deconvolute XRD intensities contributions, the broad FCC Fe (111) peak in Fig. 4b suggests a range of interplanar distances. This is confirmed by HRTEM analysis of over 40 residual catalyst particles (Fig. 5a). A histogram of lattice parameters extracted from FFT of HRTEM micrographs with lattice fringe resolution $0.2 \AA$ (Fig. 5b) shows that the distribution extends significantly beyond the equilibrium interplanar distances for FCC Fe [30] and martensite [26], evidencing substantial lattice expansion as a result of a high $\mathrm{C}$ content. Lattice parameter increases in FCC Fe nanoparticles as a consequence of $\mathrm{C}$ uptake have been observed in-situ at temperatures as low as $500^{\circ} \mathrm{C}$ [24] and extensively measured by dilatometry on bulk low-C steel samples at higher temperatures [31]. In our system, there are likely to be other contributions to the lattice expansion arising from the small size of the particles and their graphitic core-metallic shell structure. Residual thermal strain, for example, could arise from the difference between the volumetric thermal expansion (CTE) of $\mathrm{Fe}\left(\sim 10^{-6} /{ }^{\circ} \mathrm{C}\right)$ and the in-plane CTE of graphite $\left(-1.3 \times 10^{-6}\right)$.

\footnotetext{
${ }^{1}$ Field-cooled loops recorded at $10 \mathrm{~K}$ (Fig. 1a and Fig. S1b) further confirm that no antiferromagnetic $\mathrm{Fe}$ oxides are present in the samples, since the absence of exchangebias rules out the coupling between ferro- and antiferromagnetic phases.
} 

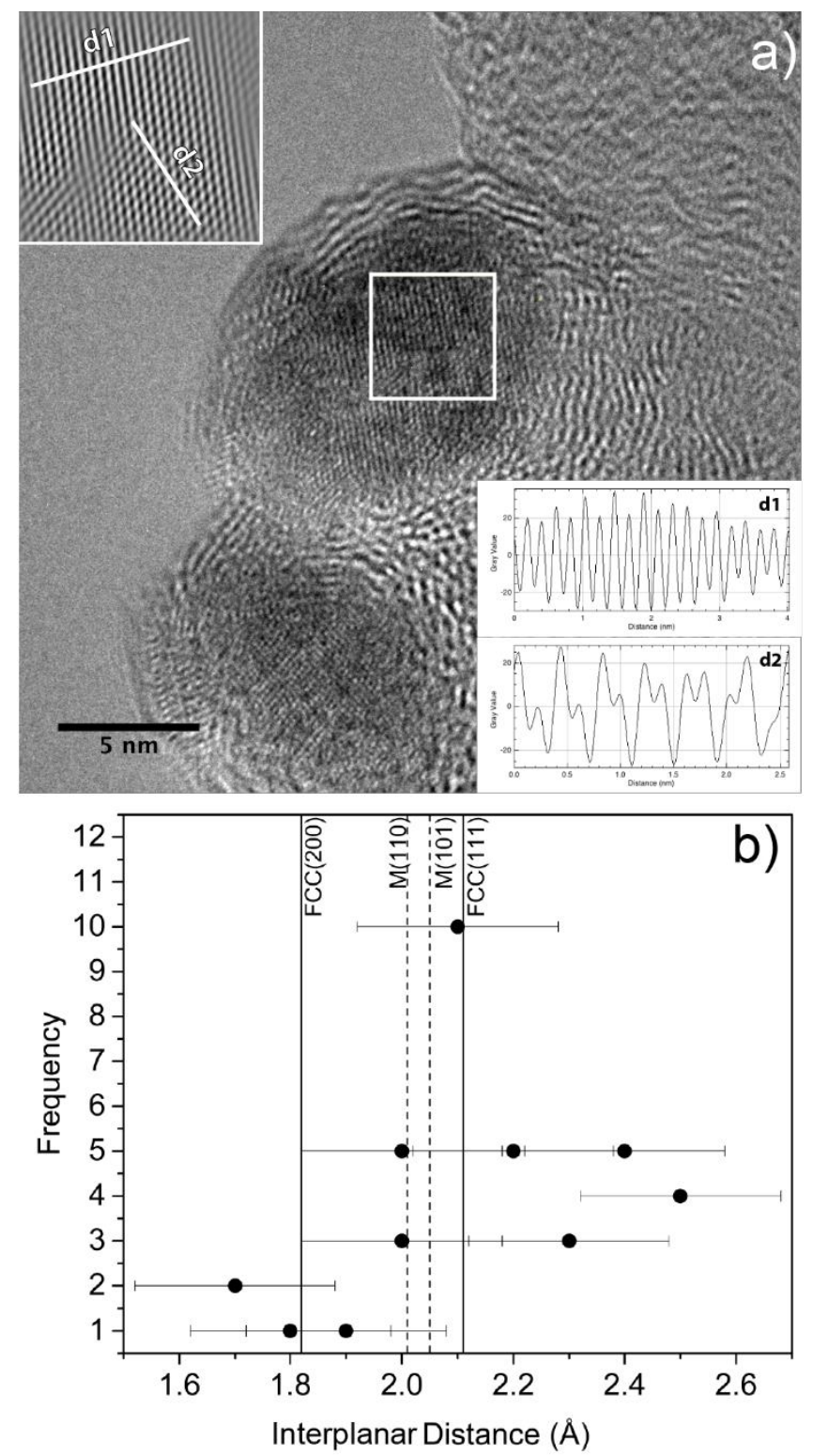

Fig. 5. (a) HRTEM image and FFT analysis of a residual catalyst nanoparticle in a CNT fibre sample. The interplanar distances measured are $\mathrm{d} 1=2.1 \pm 0.2 \AA$ and $\mathrm{d} 2=1.8 \pm 0.2 \AA$. (b) Interplanar distances distribution and the corresponding values for FCC Fe and martensite (M) with 0.77 wt. \% C content.

Finally, it is interesting to enquire into the stabilization of FCC $\mathrm{Fe}$ and martensite in the CVD growth of CNTs. Both phases form in the bulk under severe quenching and martensite can form under severe plastic deformation of perlitic steel [32]. In the CVD method used in this work nanoparticles are expected to cool down rapidly by radiative losses on account of their large surface/volume ratio, certainly 
much faster that the cooling rate extracted from the temperature profile of gases in the reaction. But quenching is unlikely to be the only cause for the stabilization of FCC Fe and martensite. Most literature reports of residual FCC Fe after CNT growth involve very slow cooling rates $\left(\sim 0.1^{\circ} \mathrm{C} / \mathrm{s}\right)$. Instead, we point to the fact that the $\mathrm{C}$ content in the nanoparticles is below the required concentration to produce a stable form of segregated $\mathrm{C}$, namely a graphitic nucleus. In Fig. S5 we plot the minimum $\mathrm{C}$ concentration needed to produce a graphitic cap as a function of Fe particle size. It includes a scheme showing the expected evolution of a $\mathrm{C}$ supersaturated Fe particle. For $6 \mathrm{~nm}$ particles, for example, this critical $\mathrm{C}$ content is as high as $10 \mathrm{wt}$. \%. The size of the particles is also too small for the formation of even ultra-fine pearlite [33] which has a lamella lateral size of around $20 \mathrm{~nm}$. In view of these considerations, it should be no surprise that only metastable retained austenite and martensite are observed in the residual catalyst produced in the continuous CNT fibers spinning process.

\section{Conclusions}

Our results show that continuous CNT fibers have ferromagnetic behavior at room temperature. The choice of promoter $(\mathrm{S}$ or $\mathrm{Se}$ ) changes the room temperature remanence from $10 \%$ to $25 \%$ and coercivity from 55 to 300 Oe. FC-ZFC curves further show that only S-CNT fibers present a clear superparamagnetic behavior below $300 \mathrm{~K}\left(\mathrm{~T}_{\mathrm{B}}=140 \mathrm{~K}\right)$, which is a consequence of the smaller size of residual catalyst nanparticles compared to those produced using Se. This is consistent with previous observations on the size and shape of catalyst nanoparticles in Se assisted CNT fiber [20] and highlights the role of the promoter in controlling $\mathrm{C}$ solubility, catalytic activity and catalyst particle coarsening through coalescence.

Ferromagnetic behavior is isotropic, arising from quasipherical residual catalyst nanoparticles. These particles are of two types: martensite with 0.77 wt. \% C content and FCC Fe. Both are encapsulated by graphitic layers. Martensite is intrinsically ferromagnetic, but bulk FCC Fe is not, therefore ferromagnetic properties of FCC Fe nanoparticles derive from severe lattice distortion due to $\mathrm{C}$ supersaturation, stabilized by the presence of the graphitic shell and their small size impeding a stable phase transformation and limiting segregation of $\mathrm{C}$ into a stable graphitic nucleus.

The experimental results of this work would suggest that FCC Fe distortion is equivalent to BCT martensite formation (Fig. S6) in terms of the resulting 
ferromagnetic properties. Further work should employ more advanced characterization techniques to confirm this hypothesis. Valuable input is likely to come from simulation work since nanoparticles produced as a product of CVD growth of CNTs are inevitably often smaller than the minimum size of an embryonic nucleus and instead resemble more a cluster of atoms [34].

\section{Acknowledgements}

Generous financial support was provided by the European Union Seventh Framework Program under grant agreements 678565 (ERC-STEM), FP7-People-Marie Curie Action-CIG (2012-322129 MUFIN), by MINECO (MT2012-37552-C03-02, MAT2015-62584-ERC, MAT2015-66888-C3-3-R, RyC-2014-15115) and by CAM MAD2D project (S2013/MIT-3007).

\section{References}

[1] V. Jourdain, C. Bichara, Current understanding of the growth of carbon nanotubes in catalytic chemical vapour deposition, Carbon. 58 (2013) 2-39. doi:10.1016/j.carbon.2013.02.046.

[2] V.L. Moruzzi, P.M. Marcus, K. Schwarz, P. Mohn, Ferromagnetic phases of bcc and fcc Fe, Co, and Ni, Phys. Rev. B. 34 (1986) 1784-1791. doi:10.1103/PhysRevB.34.1784.

[3] N. Grobert, W.K. Hsu, Y.Q. Zhu, J.P. Hare, H.W. Kroto, D.R.M. Walton, M. Terrones, H. Terrones, P. Redlich, M. Rühle, R. Escudero, F. Morales, Enhanced magnetic coercivities in Fe nanowires, Appl. Phys. Lett. 75 (1999) 3363-3365. doi:10.1063/1.125352.

[4] S. Behrens, Preparation of functional magnetic nanocomposites and hybrid materials: recent progress and future directions, Nanoscale. 3 (2011) 877-892. doi:10.1039/CONR00634C.

[5] T. Mühl, D. Elefant, A. Graff, R. Kozhuharova, A. Leonhardt, I. Mönch, M. Ritschel, P. Simon, S. Groudeva-Zotova, C.M. Schneider, Magnetic properties of aligned Fefilled carbon nanotubes, J. Appl. Phys. 93 (2003) 7894-7896. doi:10.1063/1.1557824.

[6] V.A. Labunov, A.L. Danilyuk, A.L. Prudnikava, I. Komissarov, B.G. Shulitski, C. Speisser, F. Antoni, F.L. Normand, S.L. Prischepa, Microwave absorption in nanocomposite material of magnetically functionalized carbon nanotubes, J. Appl. Phys. 112 (2012) 024302. doi:10.1063/1.4737119.

[7] U. Ritter, P. Scharff, G.E. Grechnev, V.A. Desnenko, A.V. Fedorchenko, A.S. Panfilov, Y.I. Prylutskyy, Y.A. Kolesnichenko, Structure and magnetic properties of multi-walled carbon nanotubes modified with cobalt, Carbon. 49 (2011) 44434448. doi:10.1016/j.carbon.2011.06.039.

[8] C.T. Wirth, B.C. Bayer, A.D. Gamalski, S. Esconjauregui, R.S. Weatherup, C. Ducati, C. Baehtz, J. Robertson, S. Hofmann, The Phase of Iron Catalyst Nanoparticles 
during Carbon Nanotube Growth, Chem. Mater. 24 (2012) 4633-4640. doi:10.1021/cm301402g.

[9] F.C. Dillon, A. Bajpai, A. Koós, S. Downes, Z. Aslam, N. Grobert, Tuning the magnetic properties of iron-filled carbon nanotubes, Carbon. 50 (2012) 36743681. doi:10.1016/j.carbon.2012.03.040.

[10] A.L. Danilyuk, A.L. Prudnikava, I.V. Komissarov, K.I. Yanushkevich, A. Derory, F. Le Normand, V.A. Labunov, S.L. Prischepa, Interplay between exchange interaction and magnetic anisotropy for iron based nanoparticles in aligned carbon nanotube arrays, Carbon. 68 (2014) 337-345. doi:10.1016/j.carbon.2013.11.010.

[11] J. Jiao, S. Seraphin, X. Wang, J.C. Withers, Preparation and properties of ferromagnetic carbon-coated Fe, Co, and Ni nanoparticles, J. Appl. Phys. 80 (1996) 103-108. doi:10.1063/1.362765.

[12] Bozorth R.M., Ferromagnetism, IEEE Press, 1951.

[13] J. Thomassen, F. May, B. Feldmann, M. Wuttig, H. Ibach, Magnetic live surface layers in Fe/Cu(100), Phys. Rev. Lett. 69 (1992) 3831-3834. doi:10.1103/PhysRevLett.69.3831.

[14] A. Enders, D. Repetto, T.Y. Lee, K. Kern, Perpendicular coupling and spin reorientation transition in FCC Fe/Cu/Fe trilayers, J. Magn. Magn. Mater. 272276, Supplement (2004) E959-E961. doi:10.1016/j.jmmm.2003.12.568.

[15] M. Matsui, S. Mitani, K. Doi, M. Doyama, Magnetic Anisotropy of fcc-Fe/Cu MBE Ultra Thin Film, IEEE Transl. J. Magn. Jpn. 6 (1991) 307-313. doi:10.1109/TJMJ.1991.4565155.

[16] B. Wei, M. Shima, R. Pati, S.K. Nayak, D.J. Singh, R. Ma, Y. Li, Y. Bando, S. Nasu, P.M. Ajayan, Room-Temperature Ferromagnetism in Doped Face-Centered Cubic Fe Nanoparticles, Small. 2 (2006) 804-809. doi:10.1002/smll.200500436.

[17] M. Motta, A. Moisala, I.A. Kinloch, A.H. Windle, High Performance Fibres from "Dog Bone" Carbon Nanotubes, Adv. Mater. 19 (2007) 3721-3726. doi:10.1002/adma.200700516.

[18] Y.-L. Li, I.A. Kinloch, A.H. Windle, Direct Spinning of Carbon Nanotube Fibers from Chemical Vapor Deposition Synthesis, Science. 304 (2004) 276-278. doi:10.1126/science.1094982.

[19] V. Reguero, B. Alemán, B. Mas, J.J. Vilatela, Controlling Carbon Nanotube Type in Macroscopic Fibers Synthesized by the Direct Spinning Process, Chem. Mater. 26 (2014) 3550-3557. doi:10.1021/cm501187x.

[20] B. Mas, B. Alemán, I. Dopico, I. Martin-Bragado, T. Naranjo, E.M. Pérez, J.J. Vilatela, Group 16 elements control the synthesis of continuous fibers of carbon nanotubes, Carbon. 101 (2016) 458-464. doi:10.1016/j.carbon.2016.02.005.

[21] C. Prados, P. Crespo, J.M. González, A. Hernando, J.F. Marco, R. Gancedo, N. Grobert, M. Terrones, R.M. Walton, H.W. Kroto, Hysteresis shift in Fe-filled carbon nanotubes due to gamma-Fe, Phys. Rev. B. 65 (2002) 113405. doi:10.1103/PhysRevB.65.113405.

[22] J.-I. Park, N.-J. Kang, Y.-W. Jun, S.J. Oh, H.-C. Ri, J. Cheon, Superlattice and Magnetism Directed by the Size and Shape of Nanocrystals, ChemPhysChem. 3 (2002) 543-547. doi:10.1002/1439-7641(20020617)3:6<543::AIDCPHC543>3.0.CO;2-E. 
[23] P. Tartaj, C.J. Serna, Synthesis of Monodisperse Superparamagnetic Fe/Silica Nanospherical Composites, J. Am. Chem. Soc. 125 (2003) 15754-15755. doi:10.1021/ja0380594.

[24] J. Cambedouzou, P. Landois, S. Rouzière, M. Pinault, C. Mocuta, L. Hennet, D. Thiaudière, M. Mayne-L'Hermite, P. Launois, Anomalous thermal expansion of gamma-iron nanocrystals inside multiwalled carbon nanotubes, Phys. Rev. B. 88 (2013) 081402. doi:10.1103/PhysRevB.88.081402.

[25] B. Alemán, M.M. Bernal, B. Mas, E.M. Pérez, V. Reguero, G. Xu, Y. Cui, J.J. Vilatela, Inherent predominance of high chiral angle metallic carbon nanotubes in continuous fibers grown from a molten catalyst, Nanoscale. 8 (2016) 4236-4244. doi:10.1039/C5NR07455J.

[26] Power Diffraction Card (PDF) No. 00-044-1291, (n.d.).

[27] H. Bhadeshia, R. Honeycombe, Steels: Microstructure and Properties, Butterworth-Heinemann, n.d.

[28] J. Sort, A. Concustell, E. Menéndez, S. Suriñach, M.D. Baró, J. Farran, J. Nogués, Selective generation of local ferromagnetism in austenitic stainless steel using nanoindentation, Appl. Phys. Lett. 89 (2006) 032509. doi:10.1063/1.2227827.

[29] H.K. Chow, R.L. Bogner, The Study of Retained Austenite by Means of Mössbauer Scattering Spectroscopy, in: E.L. Grove (Ed.), Dev. Appl. Spectrosc., Springer US, 1970: pp. 229-239. doi:10.1007/978-1-4684-7962-1_20.

[30] FCC Fe, Powder Diffraction Card (PDF) No. 00-052-0513, (n.d.).

[31] M. Onink, C.M. Brakman, F.D. Tichelaar, E.J. Mittemeijer, S. van der Zwaag, J.H. Root, N.B. Konyer, The lattice parameters of austenite and ferrite in Fe-C alloys as functions of carbon concentration and temperature, Scr. Metall. Mater. 29 (1993) 1011-1016. doi:10.1016/0956-716X(93)90169-S.

[32] S. Djaziri, Y. Li, G.A. Nematollahi, B. Grabowski, S. Goto, C. Kirchlechner, A. Kostka, S. Doyle, J. Neugebauer, D. Raabe, G. Dehm, Deformation-Induced Martensite: A New Paradigm for Exceptional Steels, Adv. Mater. 28 (2016) 7753-7757. doi:10.1002/adma.201601526.

[33] Y. Li, D. Raabe, M. Herbig, P.-P. Choi, S. Goto, A. Kostka, H. Yarita, C. Borchers, R. Kirchheim, Segregation Stabilizes Nanocrystalline Bulk Steel with Near Theoretical Strength, Phys. Rev. Lett. 113 (2014) 106104. doi:10.1103/PhysRevLett.113.106104.

[34] M.-C. Zhao, T. Hanamura, H. Qiu, K. Yang, Precipitation of Carbonitrides and Their Strengthening upon Non-quench Aging for Micro-alloyed Acicular Ferrite Pipeline Steels, Mater. Trans. 46 (2005) 784-789. doi:10.2320/matertrans.46.784. 